Reprod. Nutr. Dévelop., 1980, 20 (5 B), 1731-1737.

\title{
Comparaison des caprins aux ovins quant à l'ingestion, la digestibilité et la valeur alimentaire de diverses rations
}

\author{
par G. BLANCHART, J. BRUN-BELLUT, B. VIGNON \\ Laboratoire de Zootechnie, ENSAIA, \\ 38 , rue Sainte-Catherine, 54000 Nancy, France.
}

Summary. A comparative study of the intake, digestibility and nutritive value of various diets in sheep and goats.

The voluntary food intake, organic matter, crude protein and crude fiber digestibility and nitrogen balance of 23 diets have been measured simultaneously using 5 to 7 wethers and 5 to 7 castrated billy-goats in digestibility trials.

The goats ate more than the wethers (58 vs $46 \mathrm{~g} \mathrm{DM} / \mathrm{kg} \mathrm{W}^{0.75}$ ). The digestibilities obtained for goats were highly correlated with those obtained for wethers, and the mean values were similar in both species :

Organic matter :

Crude protein :

$$
\begin{gathered}
\text { digestion coefficient (CUD) goats }=1.22 \text { CUD }_{\text {wethers }}-14.5 \\
r=0.93
\end{gathered}
$$

$$
\text { digestion coefficient (CUD) goats }=1.07 \text { CUD }_{\text {wethers }}-4.8
$$

Crude fiber :

$$
r=0.94
$$

$$
\begin{gathered}
\text { digestion coefficient (CUD) } \\
r=0.78
\end{gathered}
$$

The mean nitrogen balance for goats $\left(226 \mathrm{mg} / \mathrm{kg} \mathrm{W}^{0.75}\right)$ was significantly higher than that of wethers $\left(152 \mathrm{mg} / \mathrm{kg} \mathrm{W} \mathrm{W}^{0.75}\right)$.

Based on the regression equations for each species, the calculated efficiency of proteins digestible in the intestine (PDI) was $55 \mathrm{p}$. 100, the endogenous urinary nitrogen $160 \mathrm{mg} / \mathrm{kg}$ $W^{0.75}$ and the nitrogen maintenance requirement $1.8-1.9 \mathrm{~g}$ of $\mathrm{PDI} / \mathrm{kg} \mathrm{W}^{0.75}$.

\section{Introduction.}

La méthode directe in vivo de mesure chez le mouton de la valeur alimentaire des fourrages permet de connaître leur ingestibilité et leur digestibilité. Les valeurs mesurées par cette méthode ont été jusqu'à présent appliquées à tous les ruminants. Cependant des différences entre espèces ont été mises en évidence et spécialement entre bovins ef ovins en ce qui concerne l'ingestibilité (Michalet-Doreau, 1975).

Les comparaisons entre ovins et caprins sont peu fréquentes (Geoffroy, 1974). Elles ont souvent eu comme objectif d'estimer la valeur alimentaire de fourrages particuliers tels que ceux constitués de plantes aquatiques (Heffron ef al., 1977) ou de 
plantes tropicales (Gihad, 1976) ou encore de feuillages (Wilson, 1977). Chacune de ces études portait sur un petit nombre de rations. Par ailleurs, les mesures sont souvent effectuées sur des petits nombres d'animaux à des états physiologiques différents entre espèces. L'ensemble de ces conditions explique que les résultats soient souvent contradictoires d'un auteur à l'autre.

Jang et Majumdar (1962), Chenost (1972) et Geoffroy (1974) n'ont pas mis en évidence de différence de digestibilité entre ovins et caprins, alors que d'autres auteurs considèrent que la digestibilité chez les caprins est plus forte que chez les ovins, particulièrement pour la cellulose (Jones et al., 1972 ; Devendra, 1975 ; El Hag, 1976 ; Wilson, 1977). D'autres encore montrent que la digestibilité chez les caprins est plus faible que chez les ovins pour la matière organique (Ademosum, 1970), ou la cellulose (Adeyan et al., 1975) ou les protéines (Wilson, 1975). Enfin, il n'a été réalisé que très peu de comparaisons entre ovins et caprins pour l'utilisation de l'azote (El Hag, 1976 ; Gihad, 1976).

Nous avons comparé, dans des conditions expérimentales aussi stables que possible, la consommation, la digestion ef la rétention azotée chez des boucs castrés et chez des béliers castrés, en utilisant des régimes nombreux ef variés.

\section{Matériel et méthodes.}

Nous avons utilisé des béliers castrés de race Texel et Est à laine Mérinos et des boucs castrés de race Alpine chamoisée, tous âgés d'au moins 2 ans, placés en cage à digestibilité. Chaque comparaison a porté sur des lots de 5 à 7 animaux. Chaque période de mesure a été précédée d'une phase de transition d'au moins 10 jours durant laquelle les animaux se sont adaptés au nouveau régime. Les mesures de consommation, de digestibilité et de bilan azoté ont été effectuées sur des périodes de 10 jours. Pendant la totalité d'un cycle de mesures, les animaux ont reçu leur ration à volonté (10 p. 100 de refus) individuellement, en 2 repas quotidiens.

Les analyses ont été faites sur des regroupements, par période de mesure, des échantillons représentatifs quotidiens collectifs (par lot) ou individuels de chaque aliment distribué, des refus et des fécès, après séchage à l'air à $80^{\circ} \mathrm{C}$.

Sur ces échantillons, les feneurs en cendres, en matières azotées totales (MAT) et en cellulose brute ( $C B$ ) ont été déterminées.

Sur les échantillons d'urine, recueillis pour chaque animal tous les jours ou tous les 2 jours, les teneurs en azote total et en urée (Siest, 1968) ont été mesurées.

Les caractéristiques des 23 régimes utilisés sont présentées dans le tableau 1.

\section{Résultats.}

Ingestion (fig. 1). - Sur l'ensemble des régimes étudiés, la consommation des moutons est en moyenne de $46 \mathrm{~g}$ de $M 5 / \mathrm{kg}^{P^{0}, 75}$. La dispersion des quantités ingérées entre régimes est importante $\left(\sigma=12,3 \mathrm{~g}\right.$ de $\left.\mathrm{MS} / \mathrm{kg}^{0,75}\right)$. La moyenne des consommations des boucs ( $58 \mathrm{~g}$ de $\mathrm{MS} / \mathrm{kg} \mathrm{P}^{0,75}$ ) est significativement supérieure à celle des moutons $\left(F_{(1,22)}=7,8\right)$ mais la dispersion des quantités ingérées entre régimes est identique $\left(\sigma=15,6 \mathrm{~g}\right.$ de $\left.\mathrm{MS} / \mathrm{kg} \mathrm{P}^{0,75}\right)$. 
TABLEAU 1

Nature et caractéristiques des régimes utilisés

\section{Régimes \\ (proportions en produits bruts)}

\begin{tabular}{ccc}
$\begin{array}{c}\text { Teneur } \\
\text { en }\end{array}$ & \multicolumn{2}{c}{$\begin{array}{c}\text { Composition chimique de } \\
\text { la matière sèche }\end{array}$} \\
& $\begin{array}{l}\text { Cendres } \\
(\text { p. 100) }\end{array}$ MAT $^{\text {(p. 100) }} \quad$ (p. 100)
\end{tabular}

F. luzerne (38), F. prairie (31), orge (31)

E. mais

E. mais $(97,6)$, T. soja $(2,4)$

E. maïs $(96,8)$, T. soja $(3,2)$

E. maïs $(92,6)$, T. soja $(7,4)$

F. prairie

F. prairie (50), F. luzerne (50)

F. luzerne

E. maïs $(95,4), T$. soja $(4,6)$

E. herbe

E. seigle

F. prairie (64), orge (28), T. soja (8)

E. maïs $(95,5), T$. de soja $(4,5)$

Herbe

F. de prairie (75), orge (25)

E. maïs

E. seigle $(90,9)$, blé $(6,8)$, T. soja $(2,3)$

E. seigle

E. herbe

E. seigle

E. maîs $(90,4)$, orge $(9,6)$

22 (1) E. maïs $(89,3)$, orge $(10,7)$

23

\section{6,6}

29,3

31,6

30,5

32,2

83,9

88,6

88,9

35,2

29,2

26,0

87,2

62,8

19,4

86,2

21,8

25,8

17,7

30,0

26,0

27,3

27,8

89,4

\begin{tabular}{rrr}
7,2 & 15,9 & 22,2 \\
4,1 & 7,7 & 17,3 \\
3,8 & 10,6 & 16,6 \\
4,6 & 11,7 & 16,9 \\
4,5 & 16,4 & 15,4 \\
10,7 & 6,6 & 29,8 \\
9,4 & 13,4 & 34,4 \\
9,5 & 15,2 & 34,4 \\
4,4 & 12,7 & 17,2 \\
14,4 & 13,7 & 25,7 \\
13,7 & 10,5 & 34,4 \\
6,9 & 15,2 & 20,2 \\
3,6 & 13,6 & 13,5 \\
18,0 & 23,1 & 17,6 \\
7,2 & 13,1 & 22,4 \\
5,4 & 9,7 & 19,7 \\
11,6 & 14,0 & 26,9 \\
10,9 & 10,6 & 37,4 \\
9,1 & 9,7 & 40,9 \\
7,8 & 13,2 & 26,3 \\
4,7 & 7,9 & 19,8 \\
4,8 & 7,8 & 19,6 \\
9,5 & 4,4 & 41,2 \\
\hline
\end{tabular}

(1) Le régime contenait en outre 40 ppm de Monensin.

F. : Foin ; E. : Ensilage ; T. : Tourteaux.

L'ingestion des boucs est étroitement correlée à celle des moutons $(r=0,64 * *$; $n=23$ ). La liaison n'est pas significative pour les régimes à base d'ensilage de maīs,

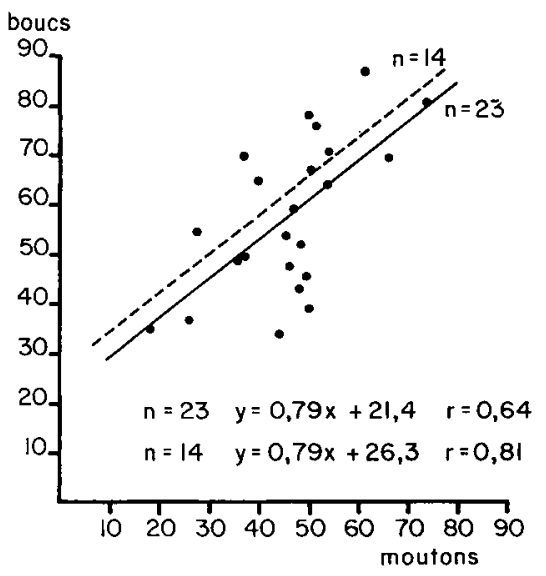

FIG. 1. - Diagramme de dispersion de la consommation entre boucs ef moutons (QI en $g$ de $M S / k g P^{0,75}$ ). 
dont les teneurs en matière sèche sont comprises entre 22 et 41 p. $100(r=0,41$; $n=9$ ) par contre elle est très étroite pour les autres rations $\left(r=0,81^{* *} ; n=14\right)$.

Digestibilité (fig. 2). - Nous n'avons pas mis en évidence de différence significative de digestibilité de la matière organique entre les deux espèces $\left(C U D_{M O}=69,8\right.$ et 70,$6 ; \sigma=6,3$ et 8,3 respectivement pour les moutons et pour les boucs), ni de différence de digestibilité des matières azotées $\left(C U D_{M A}=64,6\right.$ et 64,$4 ; \sigma=12,8$ et 14,5 respectivement pour les moutons et pour les boucs), ni de différence de digestibilité de la cellulose brute (CUD $\mathrm{CB}=58,6$ et 62,$1 ; \sigma=12,5$ ef 8,9 respectivement pour les moutons et pour les boucs).

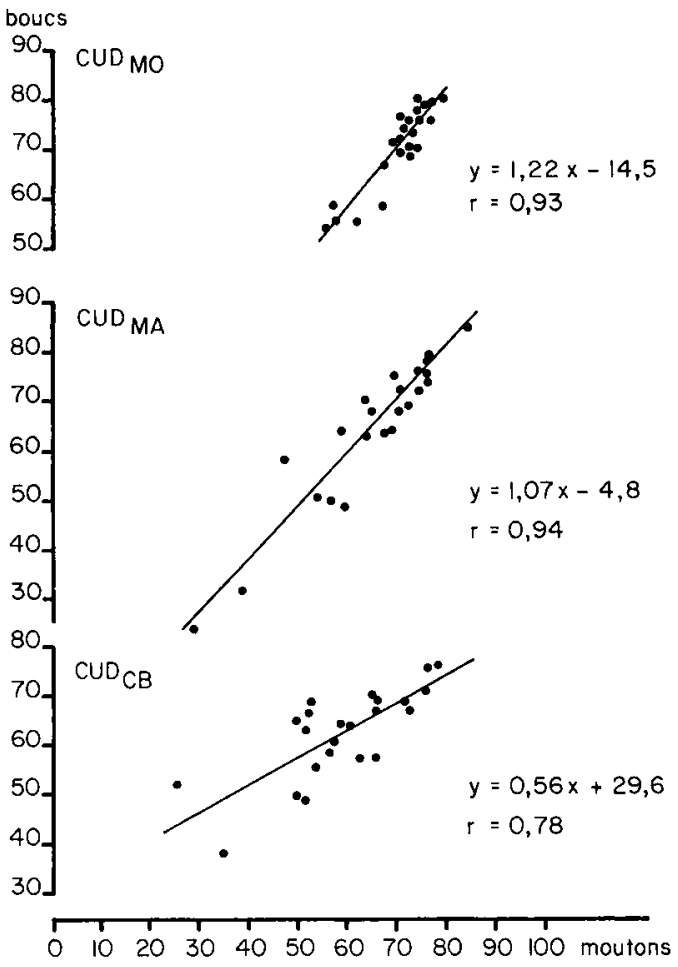

FIG. 2. - Diagrammes de dispersion des digestibilités de la matière organique, des matières azotées ef de la cellulose brufe entre boucs ef moufons pour les 23 régimes.

Il existe des liaisons très fortes entre les digestibilités mesurées sur boucs et celles mesurées sur moutons (tabl. 2).

Bilan azoté (fig. 3). - L'azote excrété par l'urine se trouve essentiellement sous forme d'urée et les variations de la teneur en azote uréique expliquent à elles seules 80 p. 100 des variations de la teneur en azote total de l'urine.

Le bilan azoté est plus élevé chez les boucs $\left(226 \mathrm{mg} / \mathrm{kg} \mathrm{P}^{\mathrm{P}, 75}\right)$ que chez les moutons $(152 \mathrm{mg} / \mathrm{kg} \mathrm{P0,75})$. L'écart entre les deux espèces $(74 \mathrm{mg} / \mathrm{kg} \mathrm{P0,75}$ en moyenne) est significativement différent de zéro. Cette différence est due à une plus grande consomma- 
TABLEAU 2

Equations de régression entre les digestibilités mesurées chez les boucs ef chez les moutons

\begin{tabular}{lllllll}
\hline & \multicolumn{1}{c}{$\begin{array}{c}\text { Equations de régression } \\
y=\mathrm{ax}+\mathrm{b}\end{array}$} & $\mathrm{n}$ & $\mathrm{r}$ & Sa & Syx \\
\hline Matière organique $\ldots$ & $\mathrm{CUD}_{\text {Boucs }}=1,22 \mathrm{CUD}_{\text {Mout. }}-1,45$ & 23 & $0,93 * *$ & 0,108 & 3,20 \\
\hline Matières azotées $\ldots .$. & $\mathrm{CUD}_{\text {Boucs }}=1,07$ CUD $_{\text {Mout. }}-4,8$ & 23 & $0,94 * *$ & 0,082 & 4,91 \\
\hline Cellulose brute $\ldots .$. & $\mathrm{CUD}_{\text {Boucs }}=0,56$ CUD $_{\text {Mout. }}+29,6$ & 23 & $0,78 * *$ & 0,097 & 5,72 \\
\hline
\end{tabular}

Seule la pente de la droite concernant la digestibilité de la cellulose brute est significativement différente de 1 ( $p<0,01)$.

tion par les boucs. La part de l'azote ingéré qui est retenue est la même chez les boucs et chez les moutons ( 34 p. 100) et la quantité d'azote fixée est étroitement corrélée entre les deux espèces $(r=0,92 * * ; n=23)$.

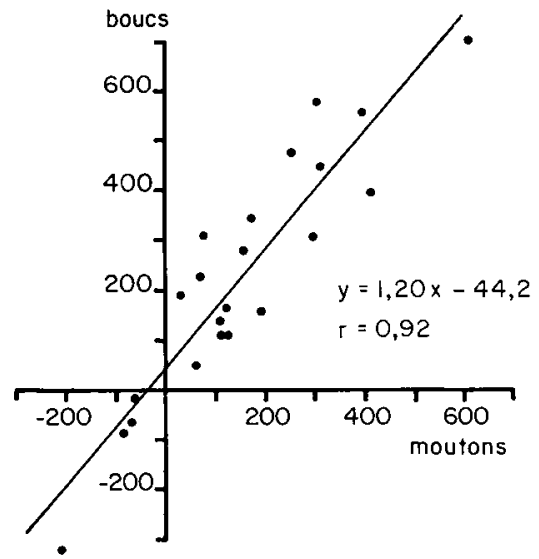

FIG. 3. - Diagramme de dispersion des bilans azotés entre boucs et moutons pour les 23 régimes

(Bilans en $\mathrm{mg} \mathrm{N} / \mathrm{kg} \mathrm{P}^{\mathrm{P}, 75}$ ).

\section{Discussion et conclusions.}

Relations entre consommation ef digestibilité. - Lorsque l'ingestibilité de la ration diminue, les boucs tendent à mieux maintenir leur consommation que les moutons (fig. 1). Au contraire la digestibilité de la matière organique des boucs tend à diminuer plus vite que celle des moutons (fig. 2). Si l'on considère les moutons comme des animaux témoins, tout se passe comme s'il existait chez les boucs une augmentation relative de la consommation, associée à une diminution relative de la digestibilité de la matière organique. Ce phénomène peut être rapproché de celui observé dans une espèce et pour une même ration, où une augmentation de la consommation s'accompagne souvent d'une baisse de la digestibilité (Church, 1971). 
Excrétion urinaire d'azote et bilan azoté. - La quantité d'azote total ef la quantité d'azote uréique excrétées dans l'urine sont étroitement liées à la consommation de matières azotées totales, de matières azotées digestibles ou de PDIN ( ${ }^{1}$ ) (tabl. 3 ).

\section{TABLEAU 3}

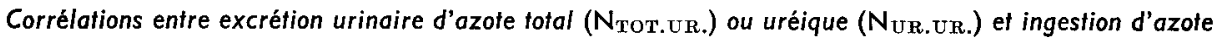

\begin{tabular}{lllll}
\hline & \multicolumn{1}{c}{$\begin{array}{c}\text { MAT } \\
\text { ingérées }\end{array}$} & $\begin{array}{c}\text { MAD } \\
\text { ingérées }\end{array}$ & $\begin{array}{c}\text { PDIN } \\
\text { ingérées }\end{array}$ \\
\hline \multirow{2}{*}{ N $_{\text {Tot.UR. }}$} & Moutons & $0,85^{* *}$ & $0,86 * *$ & $0,833^{* *}$ \\
\hline \multirow{2}{*}{ NuR.UR. } & Boucs & $0,88^{* *}$ & $0,88^{* *}$ & $0,87^{* *}$ \\
\cline { 2 - 5 } & Moutons & $0,87^{* *}$ & $0,91^{* *}$ & $0,86^{* *}$ \\
\hline & Boucs & $0,91^{* *}$ & $0,94^{* *}$ & $0,91^{* *}$ \\
\hline
\end{tabular}

L'excrétion d'azote par l'urine est aussi influencée par le niveau d'utilisation de l'azote dans le rumen. En effet, l'excès d'azote fermentescible par rapport à l'énergie disponible, exprimé par la différence PDIN - PDIE $\left({ }^{(}\right)$, est étroitement lié à l'excrétion d'azote total (moutons : $r=0,79 * *$; boucs : $r=0,75^{* *} ; n=23$ ) et d'urée (moutons : $r=0,85^{* *}$; boucs : $r=0,77^{* *} ; n=23$ ).

Dans les deux espèces, il existe une corrélation étroite entre le bilan azoté, qui caractérise la quantité d'azote fixée par les animaux, et la quantité de matières azotées digestibles ingérées :

Moutons : $y=0,41 x-107 \quad r=0,75 * * ; n=23$,

Boucs : $y=0,43 x-114 \quad r=0,80 * * n=23$,

$y=$ bilan azoté, en $\mathrm{mg} \mathrm{N} / \mathrm{kg} \mathrm{P} \mathrm{P}^{0,75}$,

$x=$ consommation de $M A D$, en $\mathrm{mg} \mathrm{N} / \mathrm{kg} \mathrm{P}^{0,75}$,

ainsi qu'entre le bilan azoté et la quantité de protéines digestibles arrivant dans l'intestin estimée par la valeur PDI ( $\left.{ }^{1}\right)$ limitante :

Moutons : $y=0,54 x-158 \quad r=0,75 * * ; n=23$,

Boucs : $y=0,55 x-166 \quad r=0,77^{* *} ; n=23$,

$y=$ bilan azoté, en $\mathrm{mg} \mathrm{N} / \mathrm{kg} \mathrm{P}^{0,75}$,

$\mathrm{x}=$ consommation de PDI limitantes, en $\mathrm{mg} \mathrm{N} / \mathrm{kg} \mathrm{P}^{0,75}$.

Ces équations permettent d'estimer l'augmentation de la fixation d'azote permise par une fourniture supplémentaire de MAD ou de PDI : globalement et sur l'ensemble des régimes éfudiés cette fixation se produit avec un rendement d'environ 42 p. 100 pour les MAD et d'environ 55 p. 100 pour les PDI.

Par extrapolation, l'exportation d'azole correspondant à la suppression de la fourniture de PDI (azote urinaire endogène) est évaluée à environ $160 \mathrm{mg} / \mathrm{kg} \mathrm{P0,75}$

(1) Protéines digestibles dans l'intestin permises par l'azote de la ration (PDIN) ou par l'énergie (PDIE) : INRA, 1978. Alimentation des ruminants. 
et correspond approximativement à la valeur trouvée par Majumdar (1960) par d'autres méthodes $(0,052 \mathrm{~g} / \mathrm{kg} \mathrm{PV})$. Enfin, les besoins d'entretien, calculés pour un bilan nul, correspondent à un apport de $1,6 \mathrm{~g}$ de $M A D / k g ~ P^{0,75}$ ou de 1,8 à $1,9 \mathrm{~g}$ de $\mathrm{PDI} / \mathrm{kg} \mathrm{P}^{0,75}$ ce qui pour un animal de $50 \mathrm{~kg}$ équivaut à $60 \mathrm{~g}$ de MAD/100 kg PV ou $70 \mathrm{~g}$ de PDI $/ 100 \mathrm{~kg}$ PV. Ces valeurs sont un peu plus faibles que celles indiquées par Fehr et Sauvant (1978) ( $80 \mathrm{~g}$ de MAD/100 kg PV ou $2,20 \mathrm{~g}$ de PDI/kg P0,75).

\section{Journées Ingestion-Digestion-Absorption de l'Association française de Nutrition, Paris, 15-16 novembre 1979.}

\section{References}

ADEMOSUM A. A., 1970. Nutritive value of Nigerian forages. 1. Digestibility of Pennisetum purpureum by sheep and goats. Niger. agric. J., 7, 19-26.

ADEYAN S. A., OGUTUGA D. B. A., ILLORI J. O., ADEGBOLA A. A., 1975. Cocoa busk in maintenance rations for sheep and goats in the tropics. Nutr. Rep. inf., 11, 351-357.

CHENOST M., 1972. Observations préliminaires sur la comparaison du potentiel digestif ef de l'appétit des caprins et des ovins en zone tropicale et en zone tempérée. Ann. Zootech., 21, 107-111.

CHURCH D. C., 1971. Digestive physiology and nutrition of ruminants. O.S.U. Book Scories, P.O. Box 489, Corvallis, Oregon 97330, U.S.A.

DEVENDRA C., 1975. The intake and digestibility of Napier grass (Pennisetum purpureum) at four, five and six weeks of growth by goats and sheep in Trinidad. Turrialba, 25, 226-231.

EL HAG G. A., 1976. A comparative study between desert goat and sheep efficiency of feed utilization. World. Rev. Anim. Prod., 12, 43-48.

FEHR P. M., SAUVANT D., 1978. Caprins, 449-467. In Alimentation des ruminanis, I.N.R.A. Publ. route de Saint-Cyr, 78000 Versailles.

GEOFFROY F., 1974. Etude comparée du comportement alimentaire et merycique de deux petits ruminants : la chèvre et le mouton. Ann. Zootech., 23, 63-73.

GIHAD E. A., 1976. Intake, digestibility and nitrogen utilization of tropical natural grass hay by goats and sheep. J. Anim. Sci., 43, 879-883.

HEFFRON C. L., REID J. T., HASCHEK W. M., FURR A. K., PARKINSON J. F., BACHE C. A., GUTENMANN W. H., St JOHN L. E. Jr, LISK D. J., 1977. Chemical composition and acceptability of aquatic plants in diets of sheep and pregnant goats. J. Anim. Sci., 45, 1166-1172.

JANG S., MAJUMDAR B. N., 1962. A study on comparative digestibilities in different species of ruminants. Ann. Biochem. exp. Med., 22, 303-308.

JONES G. M., LARSEN R. E., JAVED A. H., DONEFER E., GAUDREAU J. M., 1972. Voluntary intake and nutrient digestibility of forages by goats and sheep. J. Anim. Sci., 34, 830-838.

MAJUMDAR B. N., 1960. Studies on goat nutrition. 1. Minimum protein requirement of goats for maintenance, endogenous urinary nitrogen and metabolic faecal nitrogen excretion studies. J. Agric. Sci., 34, 329-334.

MICHALET-DOREAU B., 1975. Recherches sur les causes des variations des quantités d'ensilage d'herbe ingérées par les ruminants. Th. Doct. Ing. Univ. Nancy I, 99 pp.

SIEST G., 1968. Etude de la réaction urée-diacétylmonoxime. 2. Essais des divers adjuvants. Choix d'une méthode de dosage. Ann. Biol. clin., 26, 431-448.

WILSON A. D., LEIGH J. H., HINDLEY N. L., MULHAM W. E., 1975. Comparison of the diets of goats and sheep on a Casuarina cristata-Heterodendrum oleofolium woodland community in western New South Wales. Aust. J. exp. Agric. Anim. Husb., 15, 45-53.

WILSON A. D., 1977. The digestibility and voluntary intake of the leaves of trees and shrubs by sheep and goats. Aust. J. agric. Res., 28, 501-508. 